\title{
Deimination restores inner retinal visual function in murine demyelinating disease
}

\author{
Mabel Enriquez-Algeciras, ${ }^{1}$ Di Ding, ${ }^{1,2}$ Fabrizio G. Mastronardi, ${ }^{3}$ Robert E. Marc, ${ }^{4}$ \\ Vittorio Porciatti, ${ }^{1,5,6}$ and Sanjoy K. Bhattacharya ${ }^{1,2,5}$
}

\begin{abstract}
${ }^{1}$ Bascom Palmer Eye Institute and 2Department of Biochemistry and Molecular Biology, University of Miami, Miami, Florida, USA. ${ }^{3}$ Inceptum Research and Therapeutics Inc., Toronto, Ontario, Canada. ${ }^{4}$ Ophthalmology, University of Utah, John A. Moran Eye Center, Salt Lake City, Utah, USA. ${ }^{5}$ Neuroscience Program and ${ }^{6}$ Biomedical Engineering Program, University of Miami, Miami, Florida, USA.
\end{abstract}

\begin{abstract}
Progressive loss of visual function frequently accompanies demyelinating diseases such as multiple sclerosis (MS) and is hypothesized to be the result of damage to the axons and soma of neurons. Here, we show that dendritic impairment is also involved in these diseases. Deimination, a posttranslational modification, was reduced in the retinal ganglion cell layer of MS patients and in a transgenic mouse model of MS (ND4 mice). Reduced deimination accompanied a decrease in inner retinal function in ND4 mice, indicating loss of vision. Local restoration of deimination dramatically improved retinal function and elongation of neurites in isolated neurons. Further, neurite length was decreased by downregulation of deimination or siRNA knockdown of the export-binding protein REF, a primary target for deimination in these cells. REF localized to dendrites and bound selective mRNAs and translation machinery to promote protein synthesis. Thus, protein deimination and dendritic outgrowth play key roles in visual function and may be a general feature of demyelinating diseases.
\end{abstract}

\section{Introduction}

MS is the most common human demyelinating neurodegenerative disease, with a worldwide incidence of around $0.04 \%-0.1 \%(1-3)$. It is generally considered to be an autoimmune disease (4) caused by the breakdown of the blood-brain barrier, thereby enabling the entry of immune system components to the CNS, causing inflammation and damage. However, MS has a multifactorial etiology with a wide spectrum of clinical symptoms, and given that many other demyelinating diseases are not associated with an immune component $(3,5)$, multiple disease mechanisms are likely to be involved. Being able to decipher how complex genetic and environmental factors contribute to the inflammatory, immune, and neurodegenerative components involved in MS pathophysiology would lead to a better understanding of the disease and could lead to much needed new treatments.

Demyelinating diseases are frequently associated with optic neuritis $(\mathrm{ON})$ and vision loss (2), which is often the first clinical symptom in MS patients (6). Visual acuity measurements in MS have suggested transient loss and recovery of vision $(2,7)$; however, several studies have unequivocally shown irreversible loss of visual function and concomitant decrease in retinal nerve layer thickness (RNFL) in MS patients, irrespective of the occurrence of ON $(2,8-10)$. Although visual defects are a common and debilitating feature of demyelinating diseases, they have been studied in only a handful of transgenic mouse models (11) with only a limited analysis of vision-related parameters, and the underlying cause remains unknown. Induced transgenic (which require induction by immunogen) as well as spontaneous transgenic mouse models of demyelinating diseases and of MS have been created (as reviewed in ref. 11). However, as

Authorship note: Mabel Enriquez-Algeciras and Di Ding contributed equally to this work.

Conflict of interest: The authors have declared that no conflict of interest exists. Citation for this article: J Clin Invest. 2013;123(2):646-656. doi:10.1172/JCI64811 demyelinating diseases are multifactorial, multiple models are required to characterize all the associated contributing factors and clinical features. Pelizaeus-Merzbacher disease (PMD) presents many clinical symptoms of demyelinating diseases, such as gait abnormalities, spastic tremors, and seizures, but is not associated with autoimmunity or inflammation. It is caused by abnormal levels of myelin proteolipid protein (PLP) isoforms (12) that lead to defective growth of the myelin sheath surrounding neurons. A transgenic mouse model of demyelinating disease (ND4) was generated based on PMD by overexpression of the DM20 variant of myelin PLP $(12,13)$. These ND4 mice presented many features of both PMD and MS, including tremors and vision impairment (7). The ND4 mouse is a relatively simple model of demyelination, with no confounding immune or inflammatory factors, and thus provides an ideal system to study the molecular mechanisms underlying retinal dysfunction in demyelinating diseases.

Alterations in protein posttranslational modifications (PTMs) may contribute to some of the critical changes involved particularly in the early pathogenesis of demyelinating diseases. For example, one important and irreversible PTM is deimination, in which protein-bound arginine is converted into citrulline $(14,15)$ by one of the peptidylarginine deiminases (PADs). Hyperdeiminated regions and increased levels of the enzyme PAD2 have been found in the brains of individuals with MS $(16,17)$, and myelin basic protein (MBP) derived from MS patients is heavily deiminated (16) and shows enhanced susceptibility to proteolysis (18). However, the effect of deimination on disease pathophysiology, if any, is currently unclear.

Our investigation of deimination in the retina and brain of ND4 mice provides insight into the simultaneous occurrence of hypoand hyperdeimination in different cell types. We present analyses showing reduction in deimination in neurons commensurate with loss of inner retinal visual function and defective neurite outgrowth and identify the underlying molecular mechanism. 

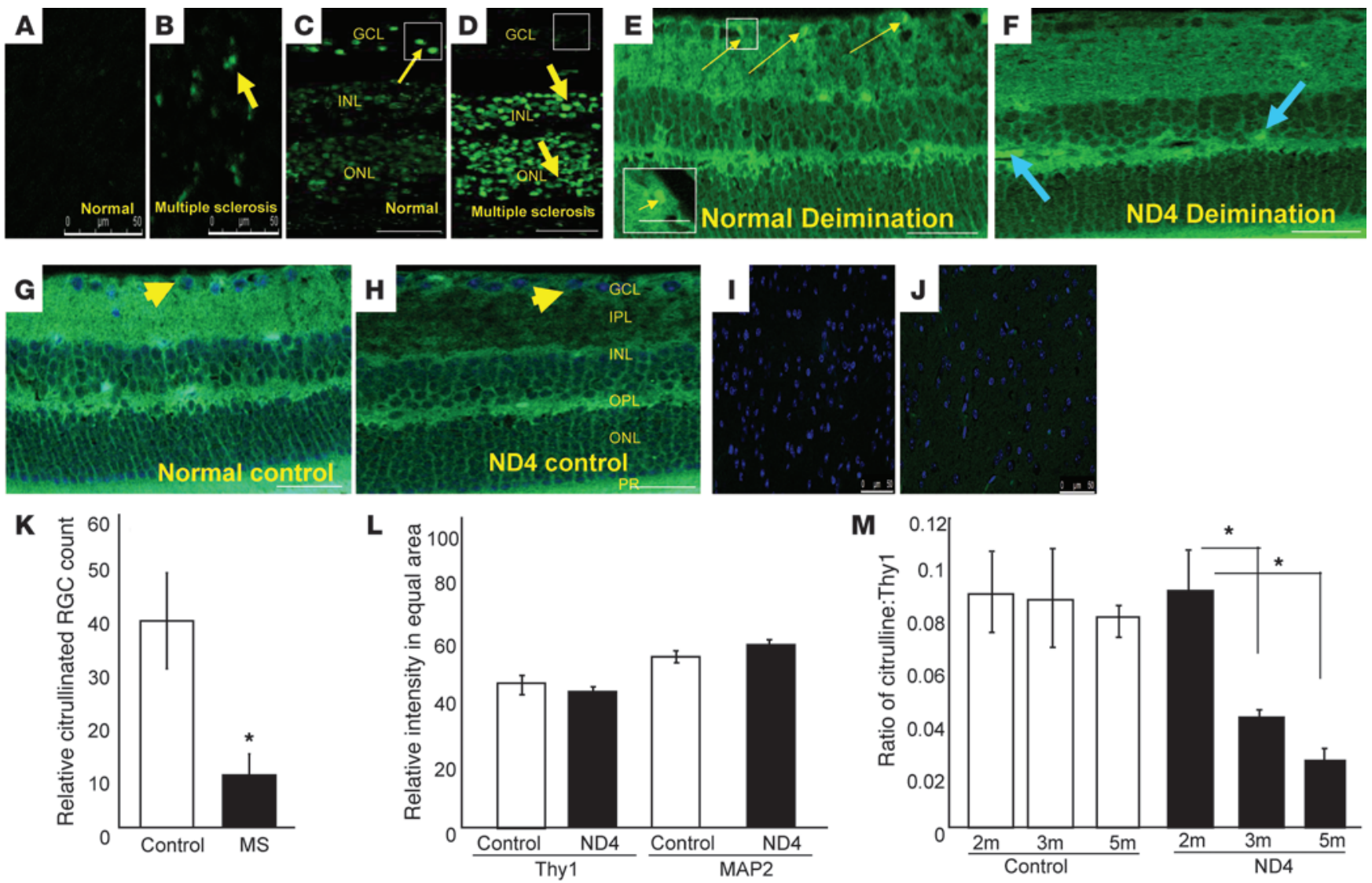

\section{Figure 1}

Relative hypodeimination in the RGC layer in demyelinating diseases. (A-M) Immunofluorescence analyses to determine deimination levels. (A and B) Representative normal and MS brains (cerebral cortex; each from a 76-year-old male of mixed European descent) subjected to monoxime treatment, using an anti-citrulline-monoxime adduct antibody (green); (C and $\mathbf{D})$ representative normal and MS human retinas treated as in $\mathbf{A}$. Thin and thick arrows indicate normal and hyperdeimination, respectively. Scale bars: $100 \mu \mathrm{m}(\mathbf{A}-\mathbf{D})$. (E) Retinal sections from normal 5-month-old control mice and (F) transgenic ND4 mice treated as in $\mathbf{A}$. Yellow thin arrows (E) indicate a subset of RGCs that is deiminated (green). Fluorescent spots (box and inset) are absent in ND4 mice (F). (G and $\mathbf{H})$ Controls without monoxime treatment subjected to anti-citrulline-monoxime adduct immunofluorescence. Blue arrows (F) indicate hyperdeiminated regions. Arrowheads show nuclei stained with DAPI (blue). GCL, ganglion cell layer; IPL, inner plexiform layer; INL, inner nuclear layer; OPL, outer plexiform layer; ONL, outer nuclear layer; PR, photoreceptor layer. Scale bars: $50 \mu \mathrm{m} ; 15 \mu \mathrm{m}$ (inset) (E-H). (I and J) Sections of control (CD1) and ND4 mice brain ( 5 month old) treated as in A. (K) Citrullinated RGC numbers in 0.1 sq. mm of human retina from 5 control and 5 MS eyes (3 regions for each eye). (L) Thy 1 and MAP 2 marker intensity in equal areas in GCL in control and ND4 mouse at 5 months of age. (M) The anti-citrulline-positive to Thy1-positive RGC cell ratio in the retina at indicated ages of ND4 and control mice (3 animals each) from equivalent regions of retina. ${ }^{*} P<0.05$.

\section{Results}

Reduced deimination in the neurons in demyelinating diseases. To determine the role of deimination in MS pathophysiology, we first analyzed the levels of deimination in different tissues from the patients with MS compared with age-matched controls. A representative patient shown here suffered from $\mathrm{ON}$ and progressive vision loss (Figure 1). The tissues were subjected to monoxime treatment to expose areas of deimination, which were visualized by immunofluorescence. In brain tissue, we detected general hyperdeiminated regions in MS as compared with normal brains (Figure 1, A and B), consistent with increased deimination of MBP as previously reported (16). However, in the eye, we found hypodeiminated regions in the retinal ganglion cell (RGC) layer (GCL), in contrast with hyperdeiminated regions primarily in the inner and outer nuclear layers and in the outer plexiform layer (Figure 1, C and D, and Supplemental Figure 5; supplemental material available online with this article; doi:10.1172/JCI64811DS1).

To study the potential role of deimination in the visual defects associated with MS, we analyzed deimination in ND4 mice, which are also visually impaired. Similar to the pattern in human MS, we observed hypodeimination of the GCL in the central retina (Figure 1, E, F, K, and Supplemental Figure 6), and hyperdeimination in the brain (Figure 1, I and J, and Supplemental Figure 5). Reduced deimination in the GCL was not associated with a general decrease in cell number (Figure 1, E and F, and Supplemental Figure 6). For example, no significant decrease in the numbers of Thy1 or MAP2 neurons was detected in either the ND4 mouse retinas (Figure 1L and Supplemental Figure 1A) or in human MS retinas (Supplemental Figure 1B 

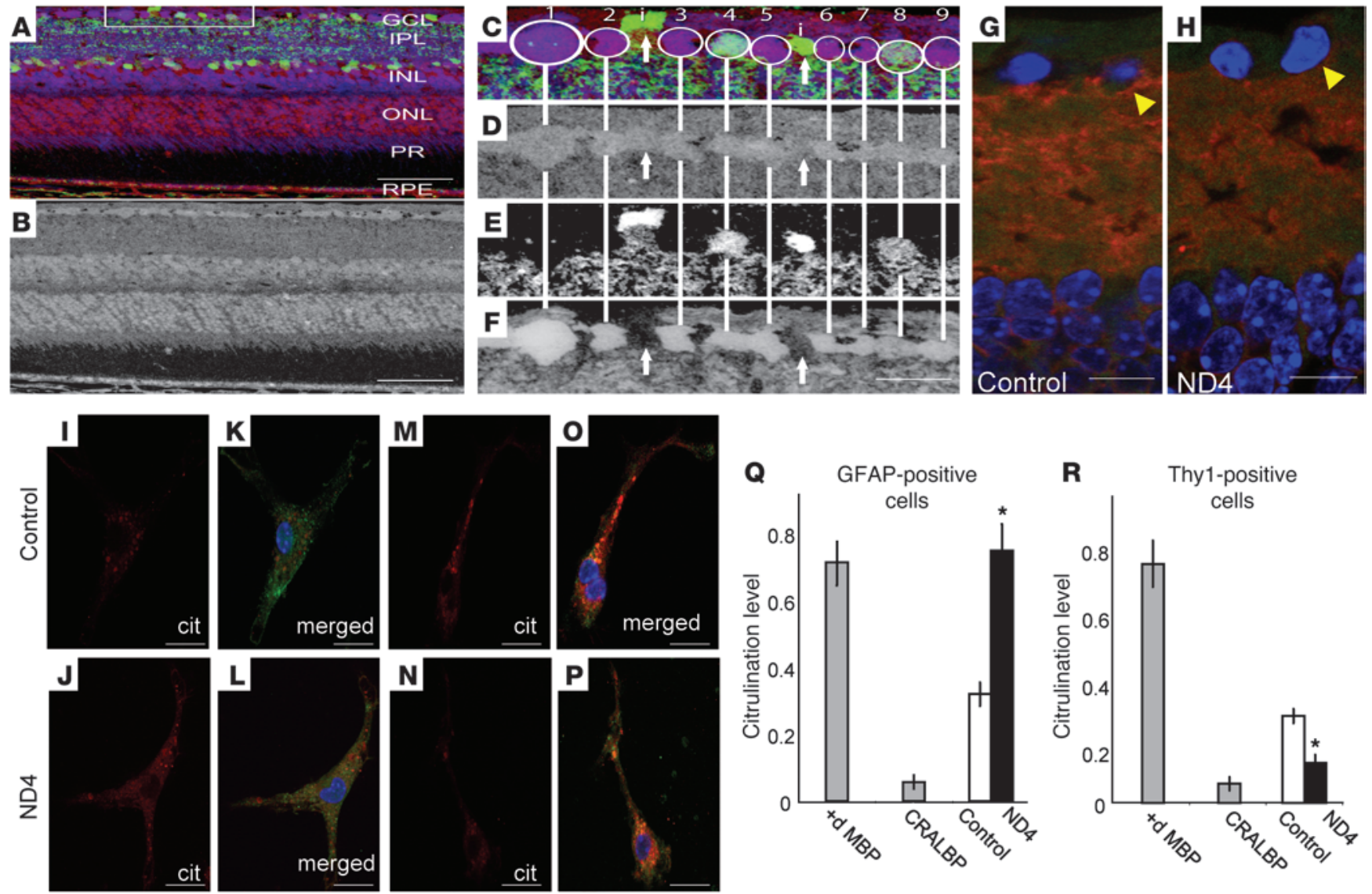

Figure 2

Decreased deimination in demyelinating diseases occurs in the neurons. (A) Validation of deimination in RGCs using small-molecule phenotyping (42) in mouse retina. Multispectral visualization of citrulline (red channel), GABA (green channel), and glutamate (blue channel). RPE, retinal pigmented epithelium. (B) Citrulline immunoreactivity in gray scale channel. Scale bars: $50 \mu \mathrm{m}$ (A, B). (C) Enlargement of the boxed region (90 $\mu \mathrm{m})$ in A. Circled cells 1-9 are RGCs with strong deimination signals. (D) Varied GABA signals. (E) Cells 4 and 8 have strong glutamate signals. (F) Cells labeled "i" (as noted in panel $\mathbf{C}$ ) are displaced amacrine cells and have weaker citrullination ( 2-fold less). Scale bar: $15 \mu \mathrm{m}(\mathbf{F})$. (G and $\mathbf{H})$ Detection of presynaptic (Syntaxin-1, red) and postsynaptic (PSD 95, green) markers in control (G) and ND4 (H) retinas (arrowhead indicates region around RGCs). Scale bars: $20 \mu \mathrm{m}$ ( $\mathbf{G}$ and $\mathbf{H}$ ). (I-L) Detection of deimination (using anti-citrulline [Cit]) in isolated astrocytes from control ( $\mathbf{I}$ and $\mathbf{K}$ ) and ND4 ( $\mathbf{J}$ and $\mathbf{L}$ ) mice. Panels $\mathbf{K}$ and $\mathbf{L}$ are the merged figure of GFAP (green) and deimination. (M-P) Detection of deimination (red) in isolated RGCs from control ( $\mathbf{M}$ and $\mathbf{O}$ ) and ND4 ( $\mathbf{N}$ and $\mathbf{P}$ ) mice. Panels $\mathbf{O}$ and $\mathbf{P}$ are the merged figure of Thy 1 (green) and deimination. Scale bars: $20 \mu \mathrm{m}(\mathbf{I}-\mathbf{P})$. (Q and R) ELISA analyses of deimination for GFAP- (Q) and Thy1-positive cells (R) from control and ND4 mice. $10 \mu \mathrm{g}$ of deiminated MBP (positive) and CRALBP (negative) used as controls. ${ }^{*} P<0.05$

and Supplemental Table 1). The observed reduction in deimination was detected within the GCL as early as 3 months of age, notably prior to the appearance of clinical symptoms (gait abnormalities and spastic tremors) (7), which began at 3.5 months (Figure 1M).

To verify that the decrease in deimination indeed occurs in RGCs, we performed additional staining of retinal sections with cell population-specific markers (amacrine cells: GABA ${ }^{+}$and GCL cells: glutamate ${ }^{+}$; Figure 2, A-F; D, E, and F represent citrulline, GABA, glutamate signals in gray scale, respectively). In the normal retina, cells harboring deiminated proteins in the GCL had a characteristic small-molecule signature (Figure 2, A-F) that is typically associated with ganglion cells (19). A weak deimination signal could occasionally be found in displaced amacrine cells of the ganglion cell layer (Figure 2, E and F). Evaluation of the areas around RGCs in ND4 retinas revealed reduced levels of the pre- and postsynaptic markers, Syntaxin-1 and PSD95, respectively (Figure 2, G and H, and Supplemental Figure 1, C-G), as well as SNAP-25 ( Supplemental Figure 1, H-J), compared with controls. The reduced density distribution of Syntaxin-1 and PSD95 suggests that in early stages of neurodegeneration in ND4 mice, processes such as dendrites emanating from RGCs may be in a state of retraction or reduced growth.

In mixed populations of isolated cells from the control CD1 mouse retina, deimination was found in both neurons and astroglial cells. In ND4 mice, isolated Thy $1^{+}$neurons had relatively little deimination, whereas astrocytes had increased levels (Figure 2, I-R).

Reinstatement of deimination improves visual function and neurite outgrowth. Like MS patients $(2,6)$, ND4 mice suffer from a loss of visual function (7) that precedes clinical symptoms of spastic tremor and gait abnormalities at approximately 3 months of age (Figure $3 \mathrm{~A}$ and Supplemental Video 1). To analyze in more detail the visu- 
A

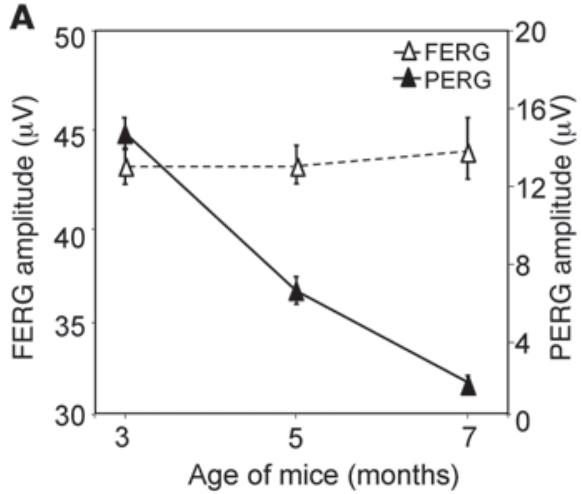

B
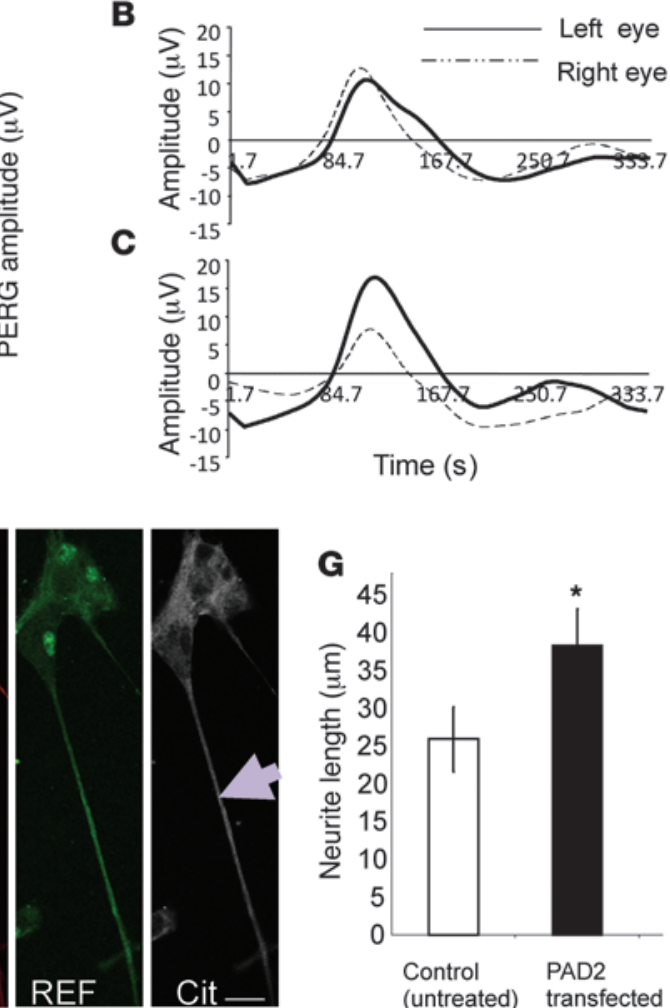

G

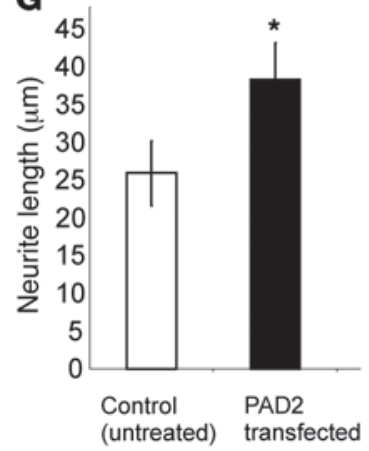

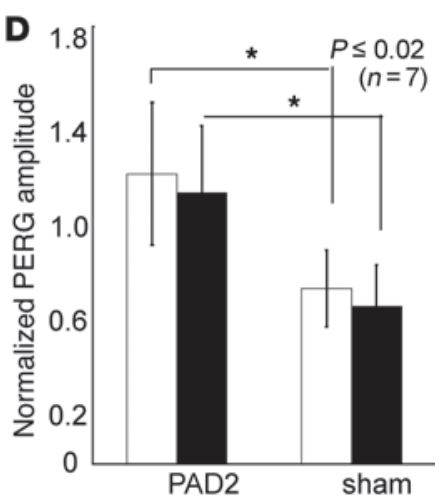

H

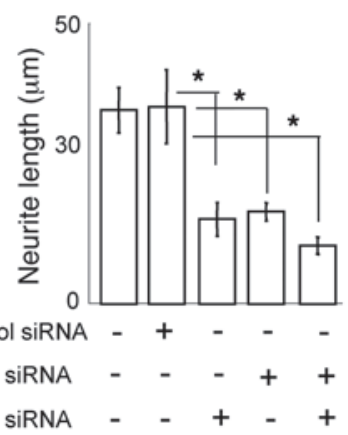

Figure 3

Restoration of deimination and visual function. (A) Evaluation of FERG and PERG amplitudes in ND4 mice. Representative mean \pm SD at indicated ages ( $n=10$ mice per group). (B-D) evaluation of visual function after PAD2 overexpression in RGCs. Representative PERG signal from an ND4 mouse at 3.5 months before injection (B) and 15 days after injection (C). Left eye: PAD2-expressing; right eye: GFP control. (D) PERG amplitude normalized to baseline for PAD2-expressing and control GFP-expressing (sham) animals for 7 injected mice at 15 days (white bars) and at 30 days (black bars). (E) Isolated RGCs from ND4 mice and (F) RGCs from ND4 mice were transfected with PAD2 lentiviral construct and incubated for identical periods. The cells were probed with anti-Thy 1 , anti-REF, and anti-citrulline as indicated. Scale bars: $10 \mu \mathrm{m}$. Arrow indicates neurite outgrowth. (G) The effects of upregulation of PAD2 on neurite outgrowth in cultured RGCs from ND4 mice, control, and PAD2transfected neurite length measurements are as indicated. $(\mathbf{H})$ Neurite length in cultured RGCs from CD1 mice upon treatment with control siRNA or siRNA against PAD2 and PAD4. In $\mathbf{G}$ and $\mathbf{H}$, mean \pm SD from 3 independent measurements (derived from 3 different animals) are shown; neurite length $(\mu \mathrm{m})$ in each experiment was average from 40 neurons. ANOVA showed a statistically significant difference between the control and treated groups. Scheffe's post hoc test showed that controls were statistically different from treated groups ( $\left.{ }^{\star} P<0.005\right)$.

al impairment seen in ND4 mice, we recorded pattern electroretinograms (PERGs), which primarily reflect the functional integrity of RGCs (20), and flash electroretinograms (FERGs), which reflect the function of retinal photoreceptors. We found that ND4 mice and their nontransgenic counterparts exhibited similar PERGs and FERGs until about 3 months of age. After this point, PERG amplitude began to decrease in ND4 mice, whereas FERG amplitudes remained the same (7).

To determine whether hypodeimination in ND4 retinas caused the associated visual impairment, we generated ND4 mice with exogenous optic nerve expression of PAD2, which is one of the enzymes that deiminates proteins and is overexpressed in the brains of MS patients. Injection of viral constructs expressing PAD2 under the control of the Thy1 or Brn3b promoter to ensure expression in the RGCs into the left eye of ND4 mice resulted in partial recovery of PERG amplitude, seen 15 days after injection (Figure 3, B and C). These PAD2-expressing ND4 mice displayed a significant gain (>30\%) in PERG amplitude compared with control ND4 mice express- ing GFP (Figure 3D). To determine whether there was reduced neurite growth associated with this visible defect, we isolated neurons from PAD2-injected and control mice and analyzed them by immunofluorescence. PAD2 expression led to a $48 \%$ increase in neurite length in isolated neurons as compared with untransfected controls (Figure 3, E-G). Consistent with this, knockdown of the principal deiminating enzymes PAD2 and PAD4 expressed in cultured RGCs using siRNAs significantly decreased neurite length compared with control siRNA in rat RGC neurons (Figure $3 \mathrm{H}$ ). A combination of PAD2 and PAD4 siRNA does not result in additive reduction compared with the reduction in neurite length achieved with treatment with siRNAs against PAD2 or PAD4 individually (Figure 3H). Similarly, PAD2/PAD4 siRNA decreased neurite length in other types of rat neurons (not shown). Thus, hypodeimination affects both neurite growth and visual function.

REF deimination is lost in ND4 mice neurons. To identify the major deiminated protein(s) in RGCs that mediate the effect on neurite outgrowth and visual function, we first performed IP of deimi- 


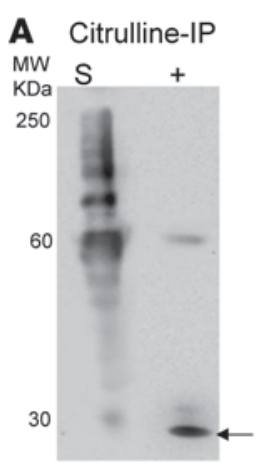

B

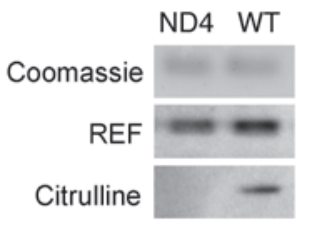

C

MADKMDMSLDDIIKLNR $\mathbb{R}^{\star} N Q R R{ }^{\star} V N R R^{\star} G G G P R R N R^{\star \star}$ PAIARGGRNRैPAPYSRPFKPLPDKWQHDLFDSG CGGGEGVETGAKLLVSNLDFGVSDADIQELFAE FGTLKKAAVDYDRSGRSLGTADVHFERRADALK AMKQYKGVPLDGRPMDIQLVASQIDPQRRPAQS GNRGGMTRSRGSGGFGGRGSQGRGRGTGRN SKQQQLSAEELDAQLDAYNARMDTS

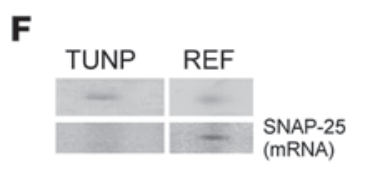

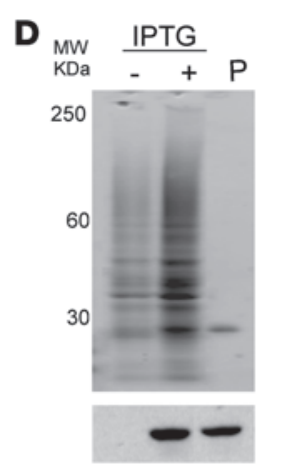
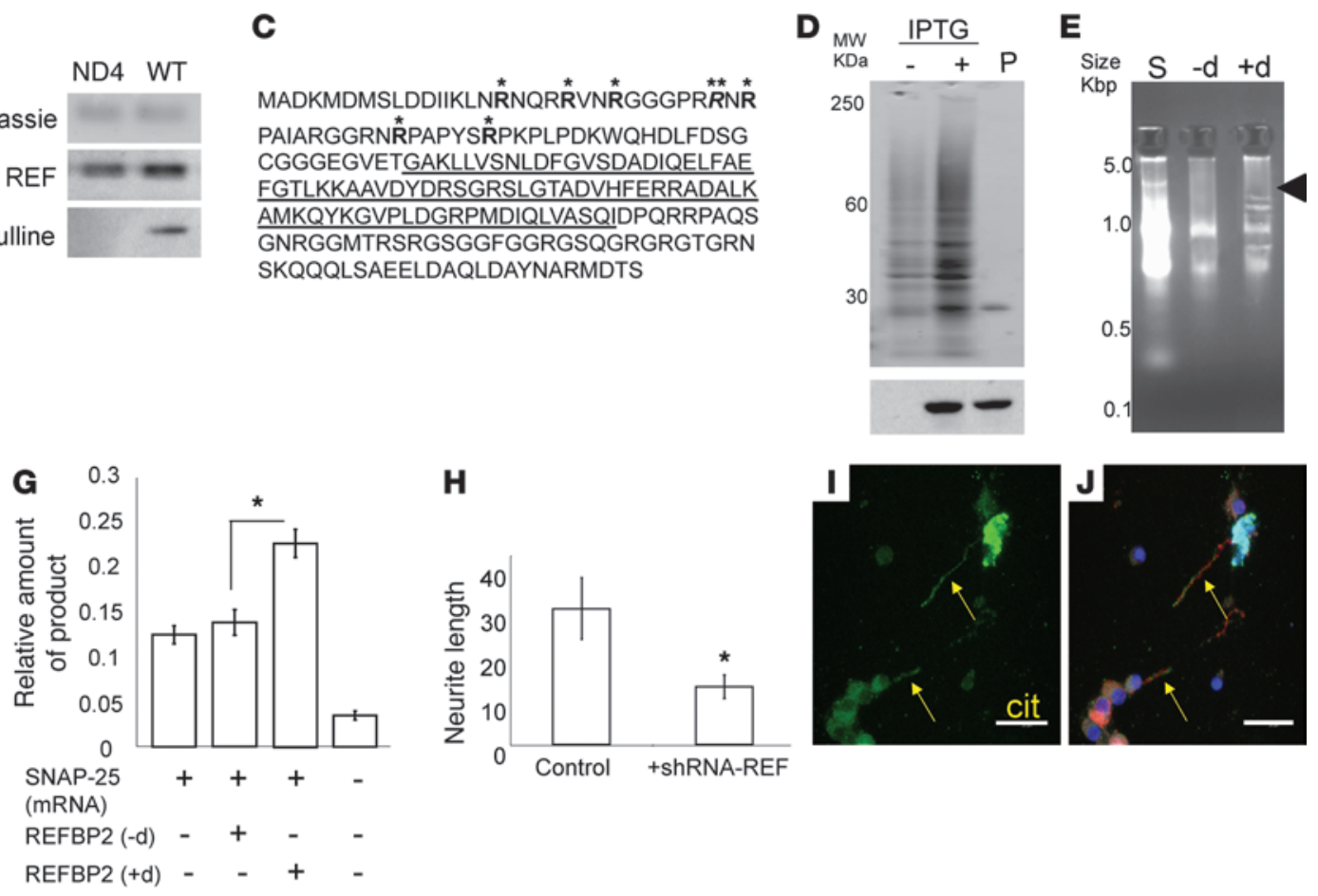
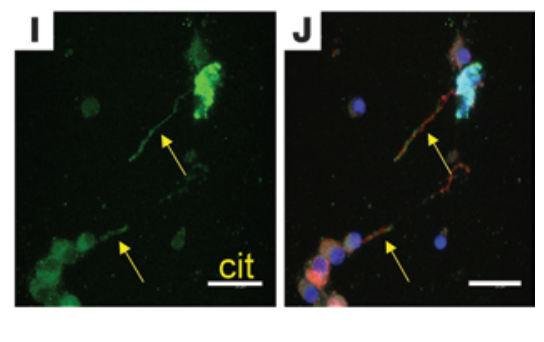

\section{Figure 4}

Identification of REFBP2 and RNA binding. (A) Western blot analysis of IP products from RGC lysates using anti-citrulline antibody. Lane 1 (S; starting material): lane 2 (+; anti-citrulline-IP). Arrow indicates the REFBP2 identification region of the gel. (B) Protein extract from RGCs were gel separated; REFBP2 ( 23 KDa) bands were excised, reloaded onto a fresh SDS-PAGE as indicated (upper panel), and detected using antiREFBP2 and anti-citrulline antibodies. (C) Mass spectrometry (LTQ OrbitrapXL) identified deiminated REFBP2 arginines (bold). The bold (*) and italicized $\left(^{* *}\right)$ arginines were found deiminated or methylated in different REFBP2 molecules. RNA binding pocket is underlined. (D) SDS-PAGE analysis of $E$. coli lysate without $(-)$ and with (+) IPTG induction and purified recombinant REFBP2 (P). Bottom panel shows Western blot analysis using an anti-His antibody. (E) RNA-binding assay analyzed on a PAGE gel in TAE buffer. Total RNA used as starting material; bound RNA species eluted from control (-d; nondeiminated) and deiminated $(+d)$ REFBP2. Arrowhead indicates enriched RNA species. (F) Purified TUNP or REFBP2 (top) was UV cross-linked with the total RNA pool and subjected to PCR for amplification of SNAP-25 mRNA (bottom). (G) Relative SNAP-25 protein amount produced by in vitro translation; $(-d)$ and $(+d)$ indicate control and deiminated REFBP2. (H) RGC neurite length upon treatment with control or shRNA-REF. Length $(\mu \mathrm{m})$ measured were averaged from 40 neurons. ${ }^{*} P<0.05$ (I) Localization of deimination (Cit) and REFBP2 in RGC neurites; (J) merged picture of DAPI (blue), anti-citrulline (green) and anti-REFBP2 (red). Arrows indicate colocalization of deimination and REFBP2. Scale bars: $25 \mu \mathrm{m}$.

nated proteins from total retinal lysates derived from normal human or control CD1 mouse eyes. Subsequent mass spectrometry consistently identified an RNA binding and export protein (REF) $(21,22)$ and its mouse homolog REFBP2 ( 24 kDa; Figure $4 \mathrm{~A})$ as the major deiminated retinal proteins. Subsequent Western blot analysis of RGC proteins from ND4 mice revealed that REFBP2 had decreased deimination compared with control mice (Figure 4B), consistent with the general loss of deimination found in the GCL by immunohistochemistry (Figure 1, C and D) and in isolated RGC cells (Figure 2, M and N). We confirmed the loss of REFBP2 deimination in ND4 mouse neurons using several different techniques. Amino acid analyses of hydrolyzed REFBP2 purified from neurons (cytosolic fraction; Supplemental Table 2) and analyses of intact mass of endogenous mouse dendritic REFBP2 (not shown) by linear mode mass spectrometry (23) were consistent with modification of up to 7 arginine residues into citrulline. High-resolution tandem mass spectrometry following pepsin digestion (Figure 4C and Supplemental Figure 2A) identified these deimination sites in REF mice that were not deiminated in ND4 mice.
To analyze whether deimination of the identified arginine residues might alter REFBP2 (Q9JJW6) structure and thus be likely to affect its function, we modeled REFBP2 using the PyMol Program (Supplemental Figure 2, D and E). The N terminus of REF has an arginine rich region, spanning residues 17-48, which may present as an RNA affinity site and may potentially interfere with mRNA binding by the bona fide RNA-binding motif of REFBP2, which spans between residues 74-154 (Figure 4C) of the protein. The deimination of $\mathrm{N}$-terminal arginines into citrulline, a neutral charge, will result in an increase of the overall negative charge in this region, thus preventing potential interference of RNA binding to bona fide RNA-binding motif by the N-terminal arginines. Another prominent structural change upon deimination relates to arginine 24 , which faces arginine 37 , forming a highly repulsive destabilizing interaction (Supplemental Figure 2, D and E). The change of arginine 24 into citrulline would be expected to render this interaction an energetically favorable one and stabilize the protein. This is consistent with experimentally observed stronger binding for selected mRNA species (23) with deiminated REF compared with controls using EMSA (described below). The 
A

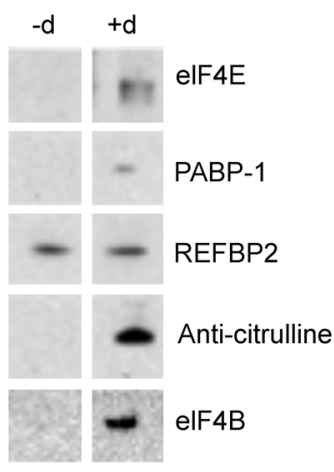

B

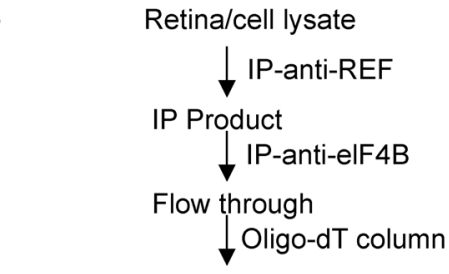

Flow through

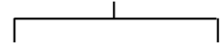

+elF4B $(0.1 \mu \mathrm{g}) \quad$ +CRALBP $(0.1 \mu \mathrm{g})$

+SNAP-25 mRNA

(250ng)

1 IP-anti-REF

IP Product

$\nabla$

1) $P C R$ for $S N A P-25$

2) WB with anti-REF
+SNAP-25 mRNA

(250ng)

$\downarrow$ IP-anti-REF

IP Product

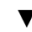

1) $P C R$ for $S N A P-25$

2) WB with anti-REF
C

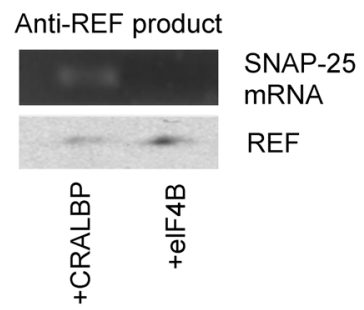

D
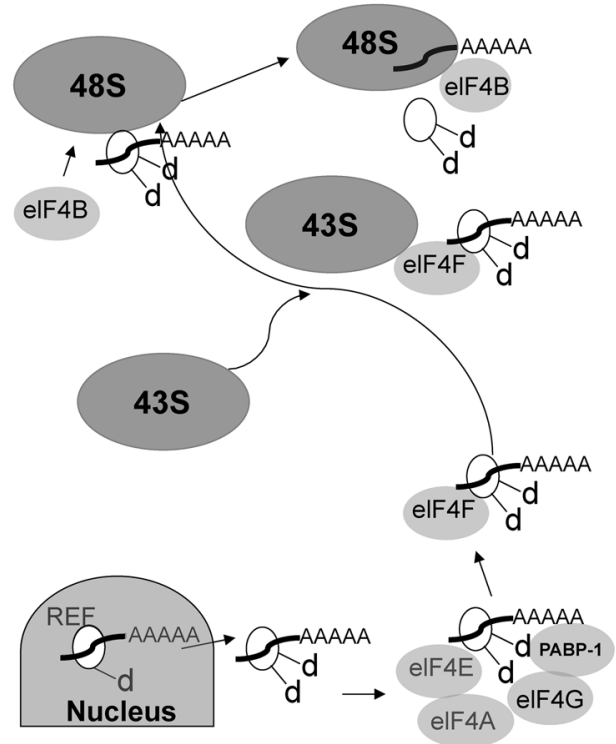

\section{Figure 5}

Effect of deimination on dendritic protein synthesis. (A) Pull-down assay with nondeiminated REFBP2 (-d) and deiminated REFBP (+d) with Western blot analyses for components of the elF4F complex. (B) A schematic diagram of the experimental paradigm of the anti-REF pull-down assay. The anti-REF IP products with elF4B removed and total mRNA were added with equal amounts of SNAP-25 mRNA in 2 equal fractions, incubated with purified elF4B or CRALBP, and subjected to second IP with anti-REF. (C) SNAP-25 amplification by PCR from the second anti-REF products (as depicted in B) with added CRALBP or elF4B. Bottom panel shows Western blot of the fractions with anti-REF. (D) Model for interaction of deiminated REFBP2 with ribosomal complex. The deiminated REFBP2 (white ellipsoid with bound polyadenylated RNA; $d$ indicates deimination) exports polyadenylated cargo from the nucleus and becomes additionally deiminated (indicated by dd). As shown by Western blot analyses in A, the deiminated REFBP2 interacts with PABP-1 and elF4E. The elF4F complex is known to interact with the 43S subunit, resulting in formation of the $48 \mathrm{~S}$ ribosomal subunit. elF4B interacts with this complex (shown by Western analysis), leading to the release of bound, polyadenylated RNA to the ribosomal complex for translation.

conversion of $\mathrm{N}$-terminal arginines into citrulline likely alters their potential interference with mRNA-binding motif, resulting in stronger RNA binding by the deiminated REF.

Deiminated REF promotes neurite outgrowth. Thus far, we have shown that hypodeimination in RGCs is associated with visual impairment and defective neurite outgrowth, and identified REFBP2 as the major deiminated protein in these cells. To determine whether REFBP2 plays a role in neurite elongation we used RNAi to knock down the expression of REFBP2. Treatment of RGCs with shRNA targeting the REF-coding region resulted in decreased neurite outgrowth compared with the control (Figure $4 \mathrm{H})$. Thus, a decrease in both deimination (Figure $3 \mathrm{H}$ ) and REF (Figure $4 \mathrm{H}$ ) reduces neurite outgrowth, supporting the regulation of neurite elongation by deiminated REF in the retina.
REFBP2 is an RNA-binding protein that plays a role in nuclear export of mRNAs (24). To determine how deimination may affect REFBP2 function in neurite outgrowth we cloned histidine-tagged REFBP2 (Figure 4, D and F) and analyzed binding to RNA. Both deiminated and nondeiminated forms of REFBP2 bound to RNA (Figure 4E); however, there were differences in the mRNA species bound (Figure 4E). Microarray analyses of the RNA bound to deiminated and nondeiminated REFBP2 identified a number of soluble NSF attachment protein receptor (SNARE) complex components (Supplemental Figure 3A; GEO GSE11843) that play a role in membrane trafficking. In particular, binding to deiminated REFBP2 in vitro resulted in enrichment of SNAP-25, VAMP2, and complexin1 mRNAs. Given that SNAP-25 has been shown to play a critical role in neurite elongation (25), we hypothesized that it 
mediates the effects of deiminated REFBP2 in the retina. Binding and enrichment of SNAP-25 mRNA by deiminated REFBP2 was further verified by UV cross-linking of protein and mRNA, and also Northern blot analysis (Figure 4F). Furthermore, histidinetagged transformation upregulated nuclear protein (TUNP), an unrelated RNA-binding protein, did not bind SNAP-25 RNA under these conditions (Figure 4F), demonstrating the specificity for deiminated REFBP2.

Deiminated REF promotes dendritic protein synthesis by interacting with the translation machinery. Since deiminated REFBP2 showed stronger binding with SNAP-25 mRNA compared with nondeiminated REFBP2 (Figure 4, E and F), we questioned whether the former affects local dendritic generation of SNAP-25 protein. We therefore determined whether binding to deiminated REFBP2 modulated the translation of SNAP-25 mRNA. Preparation of a translation-competent protein extract (free of polyadenylated RNA and SNAP-25 protein) from approximately 225,000 laser-captured RGC dendrites showed enhanced translation of added SNAP-25 mRNA (and more SNAP- 25 protein accumulation) in the presence of in vitro deiminated, recombinant REFBP2 as compared with nondeiminated REFBP2 (Figure 4G). Consistent with this, knockdown of REFBP2 in primary neurons resulted in significantly reduced levels of SNAP-25 mRNA (Supplemental Figure 3B). Biochemical studies (measured using EMSA procedure as described previously, ref. 23; filter binding assay) revealed a greater dissociation constant for SNAP-25 mRNA and nondeiminated REFBP2 compared with deiminated REFBP2, suggesting stronger binding between SNAP-25 mRNA and the deiminated protein ( 0.26 versus $0.16 \mathrm{nM})$, which is consistent with the modeled effects of deimination on REFBP2 structure shown earlier. These results demonstrate that deimination alters the RNA binding properties of REFBP2, strengthening SNAP-25 mRNA binding and enhancing protein translation of select mRNAs.

Selected species of mRNAs are found in dendrites, and there is a known role for local protein translation in maintenance and dendritic elongation (26). Consistent with a local effect on neurite outgrowth, immunofluorescence analysis detected deiminated proteins in dendrites undergoing outgrowth, and REF and deimination immunoreactivities colocalized in elongating neurites (Figure 4, I and J, and Supplemental Figure 7), supporting a deimination-based modulation of dendritic protein synthesis.

To determine whether deiminated REFBP2 interacts directly with the cellular translation machinery, we performed IP analyses of recombinant REFBP2 (deiminated or not) in fractionated cytosolic extracts from isolated RGC cells. In addition to proteins, we also evaluated whether SNAP-25 mRNA is associated with IP products of REFBP2. The IP of REFBP2 revealed that deiminated REFBP2 interacts with the translation initiation complex proteins eIF4E and PABP-1, which are part of the eIF4F complex that interacts with the $43 \mathrm{~S}$ ribosomal subunit to form the $48 \mathrm{~S}$ subunit (27), and eIF4B (Figure 5A). RT-PCR of the immunoprecipitated REFBP2-elF4F protein complex revealed the presence of SNAP-25 mRNA. However, SNAP-25 mRNA was not detected in the REFBP2 complex when elF4B was present in the precipitated complex. We therefore hypothesized that deiminated REFBP2 directs delivery of SNAP-25 mRNA to the ribosomal translational machinery (48S subunit) upon interacting with eIF4B. To test this, we immunoprecipitated the REFBP2 protein complex freed from eIF4B and combined it with equal amounts of SNAP-25 mRNA and either purified cellular retinaldehyde binding protein (CRALBP) (con- trol) or eIF4B, and then performed a second IP with anti-REF (Figure 5B). SNAP-25 mRNA was detected in the REFBP2 complex containing CRALBP but not that containing eIF4B (Figure 5C). This suggests that SNAP-25 mRNA is released upon binding with elF4B.

Our results are consistent with deiminated REFBP2-mediated delivery of SNARE complex mRNA (SNAP-25/VAMP2; GEO GSE11843) resulting in elevated local dendritic protein synthesis (28) (Figure 5D). The results also suggest that, owing to the loss of deiminated REFBP2 in MS and thereby a loss of local translation of the SNARE complex and possibly of other proteins critical for neurite elongation/outgrowth (29), neurites undergo increased disassembly and perhaps even retraction in some RGCs at an early stage of disease. The loss of deiminated REFBP2 as a carrier of SNAP-25 mRNA in pathogenic states is likely to result in decreased SNAP-25 in neuronal dendrites. Indeed, the neurites captured from freshly isolated RGCs derived from the eyes of ND4 mice had lower ratios of SNAP-25 mRNA when normalized against $\beta$-tubulin mRNA from control mice (Supplemental Figure 3C). The loss of deimination likely favors a shift in the assembly/disassembly equilibrium of dendrites toward disassembly that is mediated by the regulated synthesis of proteins involved in constitutive exocytosis/endocytosis.

\section{Discussion}

Damage to the axon and soma of neurons are well-established paradigms in many neuropathies. Here, by using a model with substantially diminished immunologic and inflammatory processes, we have instead uncovered a role for changes in the proximal parts of neurons in the damage process, which may be a general feature of progressive neuropathies.

Neurite growth and retraction has only recently been recognized as a much more dynamic and locally controlled process (28). There is growing realization that PTMs could be important regulators of local dendritic protein synthesis (29). Deimination is an irreversible PTM, and the observed loss of deimination preceded loss of visual function, suggesting that deimination is involved in some regulatory aspects that affect neuronal health. Our experimental results indicate that reduced deimination in RGCs is associated with decreased local dendritic protein synthesis, which likely leads to disassembly of neurites with impairment of neuronal connections. Relative loss of deimination in RGCs has also been detected in the myelin oligodendrocyte glycoprotein-immunized (MOGimmunized) mouse model of MS (30) as well as in glaucomatous human eyes and in the DBA/2J mouse model of pigmentary glaucoma (31). In glaucoma, axonal loss is relatively slow but progressive, and largely decoupled from inflammatory processes. Elevated intraocular pressure in glaucoma also results in RGC degeneration $(32,33)$. In addition, isolated rat ganglion cells subjected to elevated pressure (34) resulted in decreased deimination and neurite outgrowth, and RGCs subjected to centrifugal force have also shown a similar decrease in neurite outgrowth (35). In MOGimmunized mice, the reduction in deimination in RGCs has been observed prior to the clinical symptoms (M. Enriquez-Algeciras, unpublished observations). However, the kinetic analyses of deimination loss or functional visual analyses (PERG) in MOG-immunized mouse RGCs remain to be performed.

We found loss of deimination in a subset of cells in the GCL layer in ND4 mice (Figure 1E and Supplemental Figure 6). Analyses for small molecular signature suggest the cell type to be consistent with RGCs (Figure 2, A-F). The cytochemical analyses of first- 
passage, mixed-population retinal cell isolates showed none or exceedingly low levels of deimination in cells positive for the amacrine marker HPC1, but negative for RGC markers such as Thy1, $\gamma$-synuclein, or NeuN, supporting the preferential localization of deiminated proteins in RGCs. Deimination was also present in GFAP-positive cells (these may be astroglial, Müller, or retinal pigment epithelial cells, but not neurons). In ND4 mice, a decreased and elevated deimination has been observed in isolated neurons and astrocytes, respectively, (Figure 2, I-P) compared with control CD1 mice. Increased deimination in astrocytes subjected to hydrostatic pressure (34) and/or stretching (36) has been demonstrated. In our prior studies, we found that PAD2 expression and deimination, in response to pressure, is decreased in RGCs (M. EnriquezAlgeciras and D. Ding, unpublished observations) in contrast with an increase in astrocytes $(34,36)$. This opposite deimination pattern in astrocytes and RGCs was conjectured earlier (15). Insults such as hydrostatic pressure, hypoxia, and chemokines on an isolated mixed population of cells derived from CD1 mouse brain show similar increase and decrease in deimination in the astrocytes and neurons (data not shown). Presence of hypodeimination in RGCs is a likely indicator of damaged/dysfunctional neurons in the ND4 mice. In different demyelinating models, prior to manifestation of clinical symptoms of neurodegeneration, changes in the cellular microenvironment around neurons is a possibility that leads to localized insults concomitant with a reduction in deimination in neurons (Figure 2, I-P).

We hypothesize that, similar to the situation in vitro (34), elevated and reduced PAD2 expression in different cell types results in simultaneous hyper- and hypodeimination in neuronal tissues (Figure 2, I-P). However, in the retina, and in particular in the GCL, hypodeimination is more discernible due to the lack of astrocytes (Figure 1, E and F, and Supplemental Figure 6). Elevated PAD2 and consequent increased deimination in large numbers of astrocytes renders detection of hypodeimination in neurons extremely difficult in the brain. In the retina, the hyperdeiminated regions are less pronounced than in the brain and are likely contributed by retinal epithelial cells or other glial cells. A change in the deimination is also expected to alter protein-protein interactions and, as a consequence, functional biological pathways (15). It is important to note that no known enzyme exists for reversal of deimination, rendering it a long-term modification to be reversed by protein turnover. Decreased activity or failure of deiminases to act on newly synthesized proteins may also result in loss of deimination. Decreased deimination is also associated with normal aging (37), although it occurs later and at a slower rate than shown here for demyelinating disease. For example, in wild-type adult mice, a reduction in deimination begins after 6-7 months, and a $20 \%$ reduction may occur over a period of 4 months, whereas a $50 \%$ or more reduction in deimination in the GCL occurs in ND4 mice within 1 month. The deimination levels in the control and ND4 mouse are identical at 2 months of age. We would like to emphasize that both hyperdeimination (astroglial) and hypodeimination (neurons) are simultaneous processes associated with demyelination. However, the focus of the study presented here is on hypodeimination, observed in the neurons.

In ND4 mice, PERG amplitude was progressively reduced. Onset of reduction in PERG (Figure 3A) often appeared prior to manifestation of other clinical symptoms (7). PERG amplitude reflects the intactness of the connectivity of the RGCs (8). In vivo, we found decreased deimination in neurons in ND4 mice prior to onset of clinical symptoms, which was followed by a rapid decrease in PERG amplitude. The loss of deimination was manifested in isolated RGCs (Figure 2, M and O) as well as in the GCL of the retina. Conversely, the PAD2 transfection in vitro and in vivo resulted in increased dendritic growth (Figure 3, E-G) and recovery of visual functions as determined by PERG (Figure 3, B-D), which is consistent with better connectivity of RGC neurons. Reduced deimination resulting in retraction of dendrites contributing to loss of functional connectivity manifested by PERG amplitude is consistent with observed in vivo-decreased deimination and decreased PERG amplitude. In vitro, the decrease in PAD2 using siRNA resulted in decreased neurite outgrowth. PAD2 is the major deiminase in neurons and cytosolic in nature. PAD4 is predominantly nuclear and ubiquitous. The neurite length reduction occurred with both PAD2 and PAD4 siRNA. However, reduction in neurite length with 2 siRNAs in combination was not significantly different from that in single siRNA treatments. It is possible that due to considerable similarity in their sequence, the siRNA against PAD4 generated cascading intermediate inhibitory RNA that affected PAD2 mRNA expression manifested in reduction in neurite outgrowth, which is consistent with experimentally observed lack of further reduction in neurite length using combinatorial siRNA compared with singular siRNAs (Figure $3 \mathrm{H}$ ). PAD4 may compensate to a limited extent toward the overall cellular deimination in the absence of PAD2. An overall reduction in deimination partly contributed by PAD4 reduction therefore may also result in reduction in neurite outgrowth. Conversely, in vivo dendritic outgrowth will positively contribute toward PERG amplitude, consistent with increased PERG amplitude upon PAD2 injection in vivo.

In isolated neuronal cultures deiminated REFBP2 has been found to localize in the elongating neurites (Figure 4, I and J, Supplemental Figure 4, A-C, and Supplemental Figure 7), suggesting that the deiminated REFBP2 may play a role in neurite elongation. Conversely, decreased neurite length has been observed in the isolated neurons when subjected to a decrease in REFBP2 using shRNA treatment (Figure $4 \mathrm{H})$. We confirmed substantial reduction in the level of REFBP2 (Supplemental Figure 4D) following treatment with shRNA against REFBP2.

Decreased deimination in ND4 mice was commensurate with a reduction in SNAP-25 levels in RGCs (Supplemental Figure 1, H-J, and Supplemental Figure 4E). On the other hand, PAD2-injected mice demonstrated an elevated level of SNAP-25. In light of this observation of increased SNAP-25 in the retina and optic nerve (Supplemental Figure 4E), an improved synaptic transmission resulting in improvement in PERG upon PAD2 injection is a possibility that cannot be ruled out.

The results presented here are consistent with deiminated REFBP2-mediated SNAP-25 mRNA delivery in dendrites (Figure 5D). The loss of deiminated REFBP2 as an mRNA carrier in demyelinating diseases is likely to result in decreased SNAP-25 mRNA levels in neuronal dendrites. The decrease, due to a loss of local translation of these proteins that are critical for neurite outgrowth, may lead to neurite disassembly. Indeed, the neurites from ND4 mouse-derived RGCs had lower ratios of SNAP-25 mRNA (normalized against $\beta$-tubulin mRNA) compared with those from the control mice (Supplemental Figure 3C). The loss of deimination likely favors a shift toward disassembly of dendrites mediated by the negative regulation of protein synthesis. Such neurite disassembly may be one of the underlying features in the early stages of the disease. 
Many human demyelinating disorders such as MS have multiple causes and symptoms. Teasing out the individual genetic, environmental, immune, neurodegenerative, and inflammatory components is crucial for developing a detailed understanding of the pathologic process (3). We utilized here a genetic model of demyelination to distinguish neural changes largely independent of immune-activated inflammation. Our discovery of local dendritic protein synthesis regulated by deimination open up a potential new avenue for intervention strategies for development of new treatment modalities for neuropathies.

\section{Methods}

Tissue procurement, general fixation, and immunohistochemistry. The human donor MS tissue (brain and eye) was procured from Human Brain and Spinal Fluid Resource Center, VA West Los Angeles Healthcare Center (Los Angeles, California, USA). The normal and glaucomatous eyes were procured from Florida Lions Eye Bank and National Disease Research Interchange (Philadelphia, Pennsylvania, USA; details of the donors are provided in Supplemental Table 1). The original transgenic ND4 $(12,13)$ mice were procured from The Hospital for Sick Children (Toronto, Canada) as a research gift. Immunohistochemistry was performed following established procedures (37), and a kit was utilized for immunohistochemical detection of protein-bound citrulline (17-347; Millipore Corp.). Supplemental Methods includes extended experimental procedures not described here.

$I P$. For IP, protein extract was subjected to 2,3-butanedione monoxime and antipyrine treatment in an acidic environment using the citrulline kit for 10-30 minutes (Millipore Corp.), which chemically modifies the protein-bound citrulline residues for recognition by the anti-citrulline-adduct antibody. All IP experiments used no-antibody (bead only) and nonspecific antibody (anti-cochlin chicken and rabbit polyclonal) controls.

Amino acid analyses and linear mode solid state ionization mass spectrometry. We performed amino acid analyses of purified recombinant REFBP2 and recombinant in vitro deiminated REFBP2 as well as REFBP2 purified from nuclear and cytosolic fractions (from wild-type mouse; Supplemental Table 2). $25 \mu \mathrm{g}$ proteins was subjected to overnight acid hydrolysis followed by analyses on a Hitachi L-8900 amino acid analyzer following established procedures. The isolated REFBP2 protein from wild-type mouse brain was also subjected to mass spectrometry on a MALDI-TOF device (Voyager DE Pro; ABI Inc.) and analyzed in linear mode. This yielded and $m / z$ ratio of 23730.29 corresponding to unmodified REFBP2 $(\mathrm{m} / z=23730.47)$ and a large peak of 23737.32 corresponding to modification of 7 arginine residues. Relatively lower intensity ions were also recorded between 23730.29 and 23737.32, indicating the additional presence of intermediate modifications between 0 and 7 arginine residues.

Mass spectrometric identification of deiminated peptides and modeling. REFBP2 was purified from hippocampal neurons using an antibody column followed by Mono-Q and Mono-S columns employing a fast protein liquid chromatography (FPLC) system. REF protein solution $(10 \mu \mathrm{M})$ was incubated with pepsin (sequencing grade, Princeton Separations) at $37^{\circ} \mathrm{C}$ for 3 hours with an enzyme:substrate ratio of 1:50. After 3 hours, fresh pepsin was added and the digestion repeated. This process was repeated for a total of 3 additions of pepsin. Sample was then frozen until further analysis. Digestions were performed at $\mathrm{pH} 1.3$ and $\mathrm{pH}$ 2.5. For peptide identification, $2 \mu$ l of pepsin-digested REF solution was analyzed with nano HPLC ESI MS/MS interfaced with an LTQ-Orbitrap mass spectrometer (Thermo Fisher Scientific). The HPLC pump (1D Ultra; Eksigent) was configured for a "vent-load" nano HPLC experiment, and the sample was injected across a self-packed $1 \mathrm{~cm} \times 75 \mu \mathrm{m}$ trap column (IntegraFrit, New Objective/Jupiter Proteo $4 \mu \mathrm{M}$; Phenomenex) at $2 \mu \mathrm{l}$ per minute ( $20 \mu \mathrm{l}$ total). The vent was then closed and the peptides eluted across a self-packed $10 \mathrm{~cm} \times 75 \mu \mathrm{m}$ analytical column (IntegraFrit, New Objective/Jupiter Proteo $4 \mu \mathrm{M}$; Phenomenex) at a flow rate of $275 \mathrm{nl}$ per minute. Gradient elution was performed from $5 \% \mathrm{~B}\left(\mathrm{CH}_{3} \mathrm{CN}, 0.1 \% \mathrm{FA}\right)$ to $50 \%$ B over 120 minutes, and peptides were electrosprayed directly into the Orbitrap. MS/MS data were acquired in a data dependent "top 5" experiment. Because the mass shift between nonmodified and deiminated arginine is only $1 \mathrm{Da}$, the excluded width was reduced to $0.25 \mathrm{~m} / z$. The exclude list was limited to 500 , and the exclusion duration was 30 seconds. MS/MS data were then searched with MASCOT (Matrix Science) and SEQUEST (Proteome Discoverer; Thermo Fisher Scientific) against a single protein database prepared from the SwissProt database (plus random decoy), and the results were merged into Scaffold (Proteome Software). The peptides identified from the digestions at $\mathrm{pH}$ 1.3 provided coverage of $89.5 \%$ and those from $\mathrm{pH} 2.5$ provided coverage of $51 \%$. A peptide corresponding to residues $20-34$ of REF RRVNRGGGPRRNRPA (arginine residues shown in bold were identified as deiminated [citrullinated]; underlines indicate the actual peptide region captured and sequenced by mass spectrometry in several samples) was consistently identified in all pepsin digestions. A region of the spectra corresponding to RRNRPA is shown (Supplemental Figure 2C). Nondeiminated and deiminated peptides comigrated. Corresponding Y-ions of arginine residues that were deiminated were detected ( $+1 \mathrm{Da}$ as indicated) for Y-5 and Y-7 (Supplemental Figure 2B). We tried other proteases (Chymotrypsin, AspN, and LysC) for digestion of REF; however, the best results were found with 3 rounds of pepsin, each for 3 hours at $\mathrm{pH} 1.3$, which gave the best coverage and enabled identification of deiminated arginine residues in REF.

Microarray experiments. The RNA eluted from at least 2 independent binding experiments, each using recombinant and deiminated REFBP2, was subjected to cDNA preparation and 2 rounds of amplification following standard protocols and used for hybridization separately (4 different hybridization rounds) using Affymetrix Mouse Genome 430 2.0 Array Chip. Tania Contente and Elizabeth Salomon (NIH Neuroscience Microarray Consortium, Translational Genomics Research Institute [TGen], Neurogenomics Division (Phoenix, Arizona, USA) assisted with these analyses. Microarray data have been submitted to the GEO database (GSE11843).

Culture of RGCs, laser capture, and in vitro translation experiments. The rat RGCs were purified from whole retina cell suspension using established protocols (38). Briefly, the rat retinal tissue was dissociated using papain (39), and antibody-mediated plate adhesion (immunopanning) procedure (40) was used to yield a highly pure population of ganglion cells. For retina from animals older than 3 weeks, a 3-fold high concentration of BDNF was used. The 5-day-old RGCs in culture showed neurite growth and, at this stage, laser capture of the neurites was performed using Pixie III (Arcturus Inc.). Different batches of laser-captured neurites were maintained at $-80^{\circ} \mathrm{C}$ prior to being pooled for preparation of the final extract. From the 225,000 laser-captured neurites, protein extract was prepared and subjected to passage through a 3-mm microcapillary harboring oligo dT.

The mixture was used to add $0.5 \mu \mathrm{g}$ of SNAP- $25 \mathrm{mRNA}$ and a mixture of 20 amino acids to initiate translation with or without recombinant control and deiminated REFBP2. These experiments were performed following modifications of a previously published protocol for optic nerve extract (34). As controls, an identical mixture without any REFBP2 and without SNAP-25 mRNA was also used. The translation mixture was analyzed for accumulation of SNAP-25 protein. The product of in vitro translation mixture from each experiment in a total volume of $20 \mu \mathrm{l}$ per well was placed in a plate (9018 plate; Costar) and incubated for 20 minutes at room temperature. The supernatant was discarded, and the plate was washed with PBS. The plates were blocked with $1 \%$ BSA for 1 hour, washed with PBS, and incubated for 1 hour with a goat polyclonal antibody against SNAP-25 (catalog no. ab31281; Abcam Inc.) and subsequently with a. secondary antibody coupled to alkaline phosphatase for 1 hour. They were then washed with PBS, incubated 
with phosphatase substrate $(100 \mu \mathrm{l} /$ well $)$ in diethanolamine buffer, $\mathrm{pH} 7.5$, and absorbance was measured at $405 \mathrm{~nm}$ on a plate reader (Bio-tek-Synergy HT). A similar experiment was also performed with a chicken polyclonal antibody against VAMP2 (catalog no. ab14279; Abcam Inc.).

FERG and PERG recording. Methods for FERG and PERG recording have been previously described (41). Briefly, ketamine/xylazine-anesthetized mice were gently restrained with the use of a bite bar and a nose holder that allowed unobstructed vision and were kept at a constant body temperature of $37^{\circ} \mathrm{C}$ with a feedback-controlled heating pad. Eyes of anesthetized mice were typically wide open and steady, with undilated pupils pointing laterally and upward. The active electrode $(0.25-\mathrm{mm}$ diameter silver wire configured to a semicircular loop of 2-mm radius) was placed on the corneal surface by means of a micromanipulator and was positioned in such a way as to encircle the pupil without limiting the field of view. Reference and ground electrodes were stainless steel needles inserted under the skin of the scalp and tail, respectively. A small drop of balanced saline topically applied on the cornea prevented drying for the duration of recording. For PERG recording, a visual stimulus of contrast-reversing bars (field area, $50^{\circ} \times 58^{\circ}$; mean luminance, $50 \mathrm{~cd} / \mathrm{m}^{2}$; spatial frequency, $0.05 \mathrm{cyc} / \mathrm{deg}$; contrast, $98 \%$; temporal frequency, $1 \mathrm{~Hz}$ ) was aligned with the projection of the pupil at a distance of $20 \mathrm{~cm}$. Eyes were not refracted for the viewing distance given that the mouse eye has a large depth of focus because of the pinhole pupil. Retinal signals were amplified (10,000-fold) and bandpass filtered $(1-30 \mathrm{~Hz})$. Three consecutive responses to each of $600 \mathrm{con}-$ trast reversals were recorded. The responses were superimposed to check for consistency and then averaged. The PERG is a light-adapted response. To have a corresponding index of outer retinal function, a light-adapted FERG was also recorded with undilated pupils in response to strobe flashes of $20 \mathrm{~cd} / \mathrm{m}^{2} / \mathrm{s}$ superimposed on a steady background light of $12 \mathrm{~cd} / \mathrm{m}^{2}$ and presented within a Ganzfeld bowl. Averaged PERG and FERGs were analyzed to evaluate the major positive and negative waves.
Statistics. All values are given as mean \pm SD from at least 3 independent experiments. Statistical comparisons were made by 2 -tailed Student's $t$ test unless stated otherwise. $P<0.05$ was considered statistically significant.

Study approval. All animal experiments were used adhering to protocols approved by the Institutional Animal Care and Use Committee at the University of Miami. The Institutional Review Board approved/exempted the human tissues for these studies under NIH guideline category 4 .

\section{Acknowledgments}

The microarray analysis was subsidized by the NIH Neuroscience Microarray Consortium (Project: bhatt-affy581641). Tissue specimens obtained from the Human Brain and Spinal Fluid Resource Center (Los Angeles, California, USA) was sponsored by the National Institute of Neurological Disorders and Stroke/ National Institute of Mental Health, the National Multiple Sclerosis Society, and the US Department of Veterans Affairs. This work was partially supported by funds from the American Health Assistance Foundation, an unrestricted grant from Research to Prevent Blindness to the University of Miami and the University of Utah, an RPB career award (to S.K. Bhattacharya) and NIH grants P30 EY014801, EY014800, EB005832, EY02576, and EY015128, EY019077, EY016112. Microarray data have been submitted to the GEO database (GSE11843).

Received for publication May 15, 2012, and accepted in revised form November 1, 2012.

Address correspondence to: Sanjoy K. Bhattacharya, Bascom Palmer Eye Institute, 1638 NW 10th Avenue, Suite 707A, University of Miami, Miami, Florida 33136, USA. Phone: 305.482.4103; Fax: 305.326.6547; E-mail: Sbhattacharya@med.miami.edu.
1. Hagemeier K, Bruck W, Kuhlmann T. Multiple sclerosis - remyelination failure as a cause of disease progression. Histol Histopathol. 2012;27(3):277-287.

2. Kolappan M, et al. Assessing structure and function of the afferent visual pathway in multiple sclerosis and associated optic neuritis. J Neurol. 2009;256(3):305-319.

3. Lassman H. What drives disease in multiple sclerosis: Inflammation or neurodegeneration? Clin Exp Neuroimmunol. 2010;1:2-11.

4. Ziemssen T. Modulating processes within the central nervous system is central to therapeutic control of multiple sclerosis. J Neurol. 2005;5:v38-v45.

5. Popko B, Baerwald KD. Oligodendroglial response to the immune cytokine interferon gamma. Neurochem Res. 1999;24(2):331-338.

6. Xu J, Sun SW, Naismith RT, Snyder AZ, Cross AH, Song SK. Assessing optic nerve pathology with diffusion MRI: from mouse to human. NMR Biomed. 2008;21(9):928-940.

7. Enriquez-Algeciras M, et al. Evaluation of a transgenic mouse model of multiple sclerosis with noninvasive methods. Invest Ophthalmol Vis Sci. 2011;52(5):2405-2411.

8. Holder GE. Pattern electroretinography (PERG) and an integrated approach to visual pathway diagnosis. Prog Retin Eye Res. 2001;20(4):531-561.

9. Holder GE, Gale RP, Acheson JF, Robson AG. Electrodiagnostic assessment in optic nerve disease. Curr Opin Neurol. 2009;22(1):3-10.

10. Walter SD, et al. Ganglion cell loss in relation to visual disability in multiple sclerosis. Ophthalmology. 2012;119(6):1250-1257.

11. Gupta AA, Ding D, Lee RK, Levy RB, Bhattacharya SK. Spontaneous ocular and neurologic deficits in transgenic mouse models of multiple sclerosis and noninvasive investigative modalities: a review. Invest Ophthalmol Vis Sci. 2012;53(2):712-724.

12. Johnson RS, Roder JC, Riordan JR. Over-expression of the DM-20 myelin proteolipid causes central nervous system demyelination in transgenic mice. J Neurochem. 1995;64(3):967-976.

13. Mastronardi FG, Ackerley CA, Arsenault L, Roots BI, Moscarello MA. Demyelination in a transgenic mouse: a model for multiple sclerosis. J Neurosci Res. 1993;36(3):315-324.

14. Vossenaar ER, Zendman AJ, van Venrooij WJ, Pruijn GJ. PAD, a growing family of citrullinating enzymes: genes, features and involvement in disease. Bioessays. 2003;25(11):1106-1118.

15. Bhattacharya SK. Retinal deimination in aging and disease. IUBMB Life. 2009;61(5):504-509.

16. Moscarello MA, Wood DD, Ackerley C, Boulias C. Myelin in multiple sclerosis is developmentally immature. J Clin Invest. 1994;94(1):146-154.

17. Mastronardi FG, Noor A, Wood DD, Paton T, Moscarello MA. Peptidyl argininedeiminase $2 \mathrm{CpG}$ island in multiple sclerosis white matter is hypomethylated. J Neurosci Res. 2007;85(9):2006-2016.

18. Pritzker LB, Joshi S, Gowan JJ, Harauz G, Moscarello MA. Deimination of myelin basic protein. 1. Effect of deimination of arginyl residues of myelin basic protein on its structure and susceptibility to digestion by cathepsin D. Biochemistry. 2000;39(18):5374-5381.

19. Marc RE, Jones BW. Molecular phenotyping of retinal ganglion cells. J Neurosci. 2002;22(2):413-427.

20. Porciatti V. The mouse pattern electroretinogram. Doc Ophthalmol. 2007;115(3):145-153.

21. Golovanov AP, Hautbergue GM, Tintaru AM, Lian LY, Wilson SA. The solution structure of REF2I reveals interdomain interactions and regions involved in binding mRNA export factors and
RNA. RNA. 2006;12(11):1933-1948.

22. Rodrigues JP, Rode M, Gatfield D, Blencowe BJ, Carmo-Fonseca M, Izaurralde E. REF proteins mediate the export of spliced and unspliced mRNAs from the nucleus. Proc Natl Acad Sci U S A. 2001;98(3):1030-1035.

23. Ding D, Enriquez-Algeciras M, Dave KR, PerezPinzon M, Bhattacharya SK. The role of deimination in ATP5b mRNA transport in a transgenic mouse model of multiple sclerosis. EMBO Rep. 2012;13(3):230-236.

24. Stutz F, et al. REF, an evolutionary conserved family of hnRNP-like proteins, interacts with TAP/ Mex67p and participates in mRNA nuclear export. RNA. 2000;6(4):638-650.

25. Osen-Sand A, et al. Inhibition of axonal growth by SNAP-25 antisense oligonucleotides in vitro and in vivo. Nature. 1993;364(6436):445-448.

26. Bassell GJ, Singer RH, Kosik KS. Association of poly(A) mRNA with microtubules in cultured neurons. Neuron. 1994;12(3):571-582.

27. Gingras AC, Raught B, Sonenberg N. eIF4 initiation factors: effectors of mRNA recruitment to ribosomes and regulators of translation. Annu Rev Biochem. 1999;68:913-963.

28. Sutton MA, Schuman EM. Dendritic protein synthesis, synaptic plasticity, and memory. Cell. 2006;127(1):49-58.

29. Shelly M, Cancedda L, Heilshorn S, Sumbre G, Poo MM. LKB1/STRAD promotes axon initiation during neuronal polarization. Cell. 2007;129(3):565-577.

30. Sun D, et al. Myelin antigen-specific CD8+ T cells are encephalitogenic and produce severe disease in C57BL/ 6 mice. J Immunol. 2001;166(12):7579-7587.

31. John SW, et al. Essential iris atrophy, pigment dispersion, and glaucoma in DBA/2J mice. Invest $O p h$ thalmol Vis Sci. 1998;39(6):951-962. 
32. Howell GR, et al. Axons of retinal ganglion cells are insulted in the optic nerve early in DBA/2J glaucoma. J Cell Biol. 2007;179(7):1523-1537.

33. Chang EE, Goldberg JL. Glaucoma 2.0: neuroprotection, neuroregeneration, neuroenhancement. Ophthalmology. 2012;119(5):979-986.

34. Bhattacharya SK, Crabb JS, Bonilha VL, Gu X, Takahara H, Crabb JW. Proteomics implicates peptidyl arginine deiminase 2 and optic nerve citrullination in glaucoma pathogenesis. Invest Ophthalmol Vis Sci. 2006;47(6):2508-2514.

35. Kashiwagi K, Iizuka Y, Tanaka Y, Araie M, Suzuki Y, Tsukahara S. Molecular and cellular reactions of retinal ganglion cells and retinal glial cells under centrifugal force loading. Invest Ophthalmol Vis Sci. 2004;45(10):3778-3786.

36. Algeciras ME, Takahara H, Bhattacharya SK. Mechanical stretching elevates peptidyl arginine deiminase 2 expression in astrocytes. Curr Eye Res. 2008;33(11):994-1001.

37. Bhattacharya SK, Sinicrope B, Rayborn ME, Hol lyfield JG, Bonilha VL. Age-related reduction in retinal deimination levels in the F344BN rat. Aging Cell. 2008;7(3):441-444.

38. Barres BA, Silverstein BE, Corey DP, Chun LL. Immunological, morphological, and electrophysiological variation among retinal ganglion cells purified by panning. Neuron. 1988;1(9):791-803.
39. Huettner JE, Baughman RW. Primary culture of identified neurons from the visual cortex of postnatal rats. J Neurosci. 1986;6(10):3044-3060.

40. Wysocki LJ, Sato VL. "Panning" for lymphocytes: a method for cell selection. Proc Natl Acad Sci U S A. 1978;75(6):2844-2848.

41. Porciatti V, Saleh M, Nagaraju M. The pattern electroretinogram as a tool to monitor progressive retinal ganglion cell dysfunction in the DBA/2J mouse model of glaucoma. Invest Ophthalmol Vis Sci. 2007;48(2):745-751.

42. Anderson JR, et al. A computational framework for ultrastructural mapping of neural circuitry. PLoS Biol. 2009;7(3):e1000074. 